\title{
Network Management in Heterogeneous IoT Networks
}

\author{
Shunmuga Priyan Selvaraju${ }^{1}$, Ali Balador ${ }^{1,2}$, Hossein Fotouhi ${ }^{1}$, Maryam Vahabi ${ }^{1}$ and Mats Bjorkman ${ }^{1}$ \\ ${ }^{1}$ Mälardalen University, Sweden, \{shunmuga.selvaraju, hossein.fotouhi, maryam.vahabi, mats.bjorkman\}@mdh.se \\ ${ }^{2}$ RISE Research Institute of Sweden, ali.balador@ri.se
}

\begin{abstract}
Heterogeneous networks (hetnets) is an interconnection of distinctive networking paradigms to enable wider reachability and greater collaborations. In large Internet-ofThings (IoT) applications, many wireless networks are spatially co-located and intertwined forming hetnets; for instance, health monitoring devices utilising ZigBee or IEEE 802.15.4 co-exist in 2.4 GHz spectrum alongside Wi-Fi devices. Interoperability or non-obtrusive operations are required among the disjoint domains to achieve operational efficiency in overall IoT ecosystem. Specifically, network interoperability in hetnets assure desired reachability, resource orchestration and network quality. In this work, we have modelled and implemented a simulation environment for hetnets to support different schemes of network interoperability under distributed and centralised management of network. The implementation has been evaluated for network scalability and reliability to replicate large IoT hetnets. By evaluating against increasing number of nodes in the hetnet, the mean latency under distributed management is improved by 100-fold with the centralised management. Similar observations could also be made for throughput and packet loss rate.
\end{abstract}

keywords: Internet-of-Things, wireless networks, heterogeneous networks, network management

\section{INTRODUCTION}

Heterogeneous Network (Hetnet) is a paradigm for interconnected networking with various network architectures such as Wireless Sensor Network (WSN), wireless fidelity network, mobile cellular network (3G/4G/LTE/5G), wireless mesh network and vehicular network [1]. Hetnets are usually employed with large Internet-of-Things (IoT) applications like e-Healthcare, Industry 4.0 and Smart-cities. In one instance, independent domains of WSN are interconnected to form greater healthcare network where Bluetooth Low Energy (BLE) technology monitor muscular activities and IEEE 802.15.4 collect body temperature; both must work in tandem for accurate diagnosis [2]. Differences within individual domains of hetnet can occur in Medium Access Control (MAC) layer, network functions or application layer semantics. Additionally, co-located domains operating with technologies namely IEEE $802.11 \mathrm{~b} / \mathrm{g} / \mathrm{n}$, BLE, IEEE 802.15 .4 or ZigBee, coexist in the same unlicensed $2.4 \mathrm{GHz}$ spectrum. Unlicensed spectrum indicates node's unmanaged channel access and bandwidth utilisation. Even through the usage of orthogonal channels, ZigBee networks experience increased interference in presence of Wi-Fi traffic [3]. Given the collision detection mechanism such as carrier-sense multiple access, is effective only within the individual domains of wireless network.

Co-operation through networking in routing and resource orchestration can resolve above problems of interconnection between independent domains in hetnet. Specifically, network interoperability or network-domain interoperability concept achieves the aforementioned co-operation with other advantages such as multi-path routing, Inter-Domain Routing (IDR), and synchronous resource orchestrations [4]. Fig.1 depicts network interoperability between heterogeneous domains of wireless networks. Here, two prevalent technologies namely IEEE 802.11g for wireless local area network and IEEE 802.15.4 for WSN are used to represent a generic hetnet scenario. Devices utilising same technology for communication automatically constructs reachability information among themselves leading to intra-domain communication, while inter-domain communication is established through gateway which enables outward reachability with neighbouring domains. The holistic mechanism enabling inter-domain communication is referred as network interoperability. In IEEE standards, it is defined as "the ability of multiple systems or components to exchange and use the exchanged information" [5]. In other words, it is a methodological property that enables collaborations between heterogeneous domains of network. Traditionally, exterior-gateway routing protocols were able to distributively enable network interoperability through exchange of routing and reachability information [6].

Dedicated frameworks for network management or centrally managed networks can also offer interoperability. Network management can be defined as "the process of abstracting control-data plane separation in order to facilitate network programmability through a unified interface" [7]. In conventional computer networks, there exists mature management protocols such as simple network management protocol and NetConf. Recently, Software Defined Networking (SDN) has also increased the versatility of stateful network management in fifth generation (5G) cellular communications [8]. However, in IoT networks, there is an apparent lack of network management protocols except for Sensor OpenFlow [9], which is an auxiliary project of industrial framework for SDN OpenFlow. Popular WSN simulators like Contiki Cooja [10] and Mininet-WiFi [11] also appreciate the concept of network management in IoT hetnets, yet former lacks flexibility for development of actual management and later has presently mature support for Wi-Fi technology alone.

In this work, we have designed and evaluated two major network management schemes in heterogeneous networks. To be more concrete, we can classify our contributions as follows:

- Devising interoperability to enable data exchange between entities of heterogeneous network domains.

- Modelling the centralised and distributed management schemes in wireless networks.

- Developing a modular simulation environment to compare the management schemes.

- Evaluating the schemes for network performance considering network scalability and reliability.

Paper is organised as follows: section II analyses related works; section III presents main contribution including overall architecture and implementation details; section IV gives evaluation results and its implications under various schemes; and finally, Section V concludes the paper with future directions. 


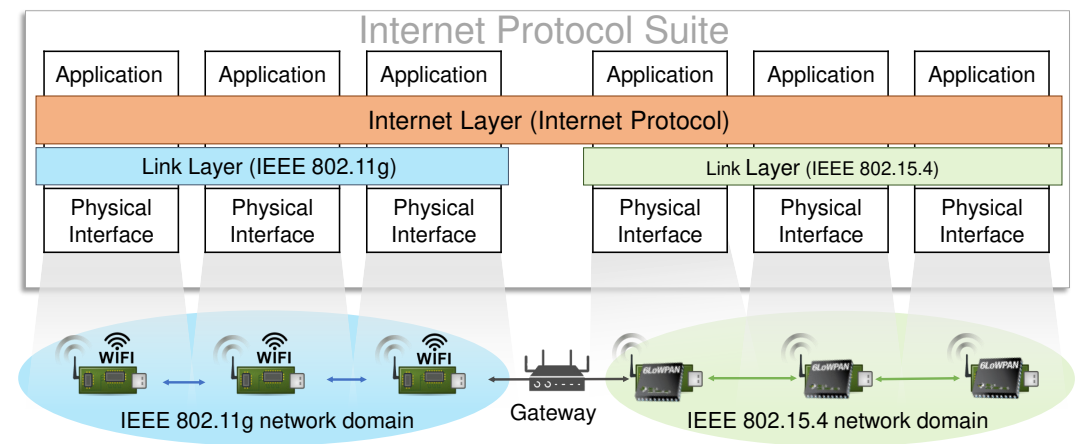

Fig. 1: An illustration of a heterogeneous IoT network, comprising IEEE 802.11g and IEEE 802.15.4

\section{RELATED WORKS}

With advent of $5 \mathrm{G}$, there have been several proposals for lossless service transition between super-high frequency and on-premise networks. These proposals have been promoting network interoperability as holistic management over indooroutdoor domains. From the proposals, term "network interoperability" is also referred as holistic management, intertechnology communication, vertical handovers, and interdomain networking in different networking paradigms [8][12]. In this section, we review some of the relevant papers with respect to application and suitability for IoT hetnets.

Distributed Mobility Management Architecture (DMMA) has been devised in [12] that performs vertical handovers and inter-domain networking between heterogeneous networks such as Wi-Fi technologies and cellular technologies - 4G and 5G. It enables communication between SDN controlled heterogeneous domains based on distributed hash tables. The architecture has considered high level of abstraction for monitoring network operations and the abstraction is vaguely technology-generic, which has mostly ignored the considerations of low-power radios like IEEE 802.15.4.

In [13], heterogeneous domains controlled by SDN controller in IoT infrastructure has been grouped into homogeneous groups of SDN domains promoting interoperability through uniformity in information exchange. They improve security of the system by prioritising and adhering to the Quality-of-Service (QoS) of critical flows for security compliance and updates. The proposal has robust operation in low link-error environments, unlike Low-power Lossy Networks (LLNs). Additionally, the presented architecture elaborates the QoS of security considerations in heterogeneous networks while disregarding scalability and reliability aspects, which are also important requirements of Massive IoT.

Interestingly the solution in [14] counters the heterogeneity in inter-technology communication between IoT simulators through usage of Linux tools, similar to the contribution of our work. Adapters have been implemented utilising virtual interfaces, which interconnects different network simulators such as ns-3, OMNET++ and Mininet-Wifi. Currently, the work has been presented as a preliminary implementation without enabling routing and network functions across multiples adapters. This makes the framework well-suited for creation of interconnections between heterogeneous domains, while lacking the capability for network management.

Some frameworks are specialised in targeting network interoperability through inter-network management. Likewise,
ORCHESTRA [8] framework performs network management with abilities of load balancing, packet replication, flow scheduling, per-packet and diligent handovers in 5G hetnets. The framework performs with usage of open abstracted MAC layer for each network, and centralised pseudo-SDN controller. Though it has been envisioned for comprehensive or holistic operation among hetnets, it currently focuses only on the aspect of edge router and gateway operation.

Moving away from $5 \mathrm{G}$ and considering interoperabilitybased solutions in constrained networks or specifically WSN, SDN promotes multi-tenancy in WSN and robust interface for coupling with exterior-gateway networks. Soft-WSN is a softwarized network management, which can offer multitenancy through policy-driven device or topology management in WSN [15]. With SDN, programmable networking optimises the operations of edge router for efficient interconnection between heterogeneous networks. Framework for synchronous management between SDN-based domains of local WSN, core network and cloud datacentres has been developed in [16]. While easing the interface between constrained network and regular Internet, SDN-based proposals offer solutions for IDR within WSN yet lack comprehensive management for QoS and scalability.

Many recent works have utilised SDN as their key aspect for inter-domain networking. Network interoperability through SDN in IoT hetnets is an attractive approach, but SDN implementation in constrained networks faces the burden of need for low-complexity operations. In turn, the constraint for lowcomplexity compromises the flexibility and openness of the solution to collaborate with other SDN-based solutions, which can create new groups of disjoint SDN domains. Thus, in this work, we present the development of a low-complex network management solutions to firstly study the perils of large IoT hetnets. The study aids in assessing design constraints before actual development of network management in future IoT hetnets.

\section{INTEROPERABILITY FOCUSED NETWORK MANAGEMENTS}

We define the network interoperability as a property of network management to enable collaboration, interaction, and provision of wider reachability among different network domains. It can be performed through centralised or distributed network management. In these schemes, logical location of the manager impacts the performance and operations. Centralised architecture gives the advantage of consolidating the entire network operations under a single viewpoint, and also the 
manager is facilitated inside a dedicated network host. However, centralised setup demands a dedicated and robust control channel that causes significant traffic overhead. Additionally, there is the vulnerability of single point-of-failure in control plane. Distributed architecture organises network management over a selective group of nodes. These nodes are independent; hence the failure of some nodes does not cease the operation of the network. However, there is an inherent difficulty in consolidating the control information across the network, as with propagation of policy and its network-wide conformation. The framework also needs to consider the allocation of network resources and isolation of network services within the participating nodes.

In this work, we have developed a simulation environment to emulate and test the different schemes of network management performing network interoperability. Comparatively testing the schemes in IoT hetnets helps in identifying the features and drawbacks for each of them. Further, we elaborate the architecture and implementation of management schemes.

\section{A. Architectures of network management}

The environment is composed of simulated domains of IoT hetnet namely IEEE 802.11g and IEEE 802.15.4 networks which are interconnected through a virtual gateway. The functionalities of these domains include emulation of individual nodes; implementation of separate IP suite for each node; and facilitation of software-based interconnection and network management among the nodes. Fig. 2 presents the schematic view of simulation environment along with routing graphs under the management schemes. Each network domain contains 30 nodes and 1 edge node. Edge nodes or node bordering external network are Access Point (AP) of IEEE $802.11 \mathrm{~g}$ domain and Root Node (RN) of IEEE 802.15.4 domain, in a grid topology. Both AP and RN are the focal points of communications in their respective domains, as they interconnect interior and exterior networks through the virtual gateway with Ethernet connections. They are also multi-homed nodes, which means the nodes are capable of communication and networking in more than one technology. Specifically, AP diverts the IP packets from IEEE $802.11 \mathrm{~g}$ frames to Ethernet frames and vice versa, whereas RN performs the same for IEEE 802.15.4 and Ethernet frames.

In the architecture, network interoperability is enabled between IEEE 802.11g and IEEE 802.15.4 network domains through exchange of routing or reachability information. Edge nodes AP and RN hold the routing information for external domains - IEEE 802.15.4 and IEEE 802.11g respectively. The management schemes perform network interoperability by essentially optimising the routing and resource orchestration towards network edge within each domain. In distributed scheme, every node route and orchestrate network resource towards edge node unaware of the neighbouring domains, which disregards prioritised resource orchestration for inter-domain networking. In contrast, centralised management organises the nodes to specially orchestrate the network resources for interdomain networking. This is depicted in Fig.2, the routing graph is accessible and updated commonly by every node under distributed management without any prioritisation, but centralised management enables only the nodes involved in the actual data communication.

Two management schemes - distributed and centralised, are devised using mesh routing and virtual network manager respectively. The development of distributed management utilises available mesh routing protocols with appropriate adoptions and synchronous operation in respective network domains. The distributed management allows independent network discovery and maintenance among the nodes. Specifically, in IEEE 802.15.4 network, distributed scheme is performed by Routing Protocol for LLN (RPL) that orchestrates the network structures by generating a Destination Oriented Directed Acyclic Graph (DODAG) at the RN [17]. This DODAG graph is utilised to select parents and perform routing by each node. In IEEE $802.11 \mathrm{~g}$, mesh standard IEEE $802.11 \mathrm{~s}$ defines a self-configuring architecture through broadcast of radio parameters over multi-hop topology in MAC layer [18]. Individual mesh-station's beacon carries the information to join the mesh and perform routing in the network. In general, routing is performed for finding the path towards $\mathrm{RN}$ in IEEE 802.15.4 and AP in IEEE 802.11g domains, which connects to neighbouring domains.

The development of centralised management utilises virtual manager in which the network manager is considered to be virtually implanting the networking rules in the nodes. Currently virtual mechanisms are used, as appropriate tools are unavailable for development [9]. Thus, simplified route calculation and uniform hard coding approaches have been used to emulate the virtual functionalities of central manager and control channel respectively. Under centralised network management, all nodes remain inactive, and only the selective nodes are instructed to communicate. The centralised management provides the network control based on consolidated network information and holistic view of the network. From the example configuration shown in the Fig.2, only selective nodes, that is, the farthest nodes are allowed to communicate and construct the routing graph.

In spatially co-located heterogeneous wireless domains, the transmission and interference patterns of individual nodes overlap increasing the complexity in modelling the propagation medium. The realistic co-existence aspect of the simulation environment needs to be implemented with supervised machine learning techniques to generate a good predictive model. Nevertheless the evaluation of link quality in current setup is based on simplistic propagation model that accounts for channel usage or bandwidth utilisation of the nodes alone. Through the simplistic propagation model, we introduce Gaussian noise or randomly generated packet drop rate at the nodes for replicating non-deterministic behaviour. Simulation of packet drop in the channel can determine the robustness of different management schemes.

\section{B. Implementation}

Linux networking tools have been mainly used to build the simulation environment, especially the concepts of Control group (Cgroup), network namespaces and virtual networking [19]. The Cgroup reserve dedicated network resources and namespace concept assures accurate routing for independent operations of each simulated node. The virtual networking is the main process of generating logical interfaces inside Linux kernel to replicate actual wireless interface. Virtual interfaces are interconnected by UNIX pipes, isolated from physical environment and so, randomised packet drops are generated to simulate realistic medium through traffic shaping tool tc. When a node is set to drop packets, it simply turns off the 

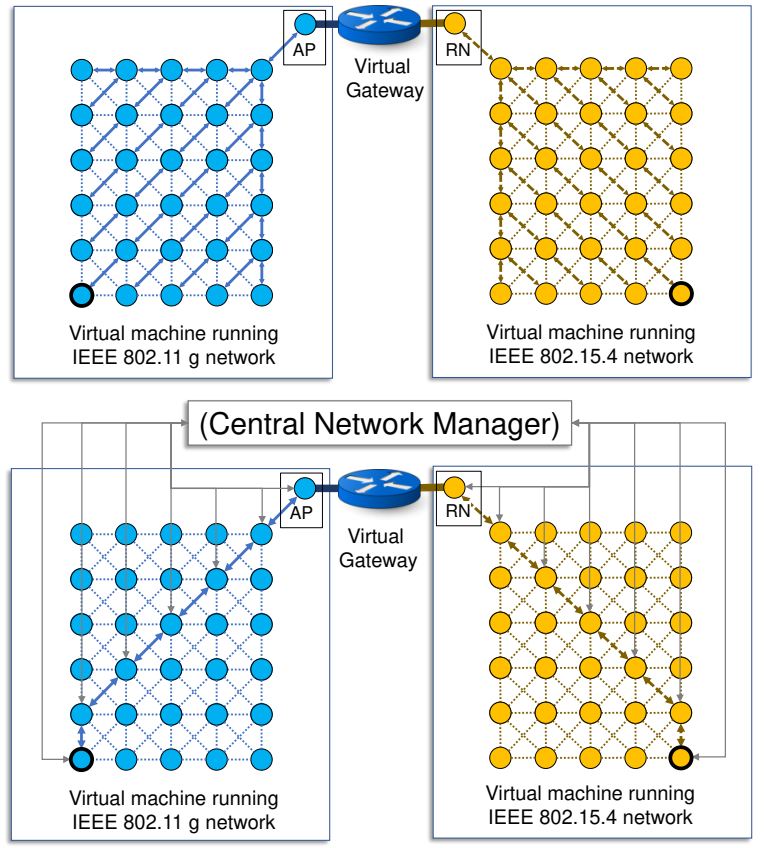

Types of links and nodes: $\quad \longrightarrow$ IPv4/IEEE 802.11g $\quad$ IPv4/Ethernet $\longleftrightarrow$ (Control Channel) $\rightarrow-\rightarrow$ IPv6/IEEE 802.15.4 IPv6/Ethernet

O Communicating nodes

Fig. 2: Schematic view of the simulation environment and the routing graphs under different network management schemes: (i) distributed management (top); and (ii) centralised management (below).

radio interface which mimics the characteristic of duty-cycling in the node.

We have built the simulation environment using three VirtualBox VMs for each IEEE 802.11g and IEEE 802.15.4 network domain along with one for gateway. All the VMs are interconnected using virtual local area network switch. Selected configuration of the VMs is as follows: Linux 5.4.0 kernel, Debian Ubuntu 20.04, 3 GB RAM, 2 core CPU at $2.60 \mathrm{GHz}$ and 1 Gigabit Ethernet interface. IEEE $802.11 \mathrm{~g}$ and IEEE 802.15.4 networks use IPv4 and IPv6 as internet layer of IP suite respectively. Incidentally, IPv4 networking is performed between the gateway and IEEE 802.11g VM; and IPv6 networking is performed between the gateway and IEEE 802.15.4 VM. Given the varying Internet layers, protocol translation is done at the gateway using Jool tool which performs network address translation between IPv4 and IPv6 networks using explicit address-mapping table.

Inside the simulation, every node is located in identical radio environment. IEEE $802.11 \mathrm{~g}$ domain uses the channel 5, and IEEE 802.15.4 domain uses the channel 13. Within the IEEE 802.15.4 domain of the simulation environment, each node receives a dedicated virtual IEEE 802.15.4 interface that emulates a physical radio transceiver to produce network traffic among them. Each IEEE 802.11g nodes receives a dedicated virtual IEEE 802.11 interface. Edge nodes, AP and $\mathrm{RN}$, receive an additional virtual Ethernet interface to establish external connection with the gateway between the heterogeneous domains. In the base simulation environment, Mininet-wifi is used to simulate IEEE 802.11g network, as it offers robust and scalable support for IEEE $802.11 \mathrm{~g}$ technology. However, the interface and support for IEEE 802.15.4 in Mininet-wifi is still in nascent stage without any implementation for mesh routing. Thus, IEEE 802.15.4 nodes are simulated using Linux mac802154_hwsim for MAC implementation, and Linux iwpan tools to implement radio links.

Routing information to enable network interoperability are installed using stateful network rules with the help of Linux ip route. These rules enable static routing between the edge nodes AP and RN and the gateway. The distributed management is implemented using IEEE 802.11 s of Mininet-wifi with path selection mechanism from derived tree-oriented Adhoc On Demand Distance Vector (AODV) protocol; and RPL using linux-wpan rpld toolkit with path selection from storingmode operation or stateful routing table for upward and downward routing. The distributed implementation utilises MAC layer or layer 2 as data-forwarding plane in IEEE $802.11 \mathrm{~g}$ domain and internet layer or layer 3 as data-forwarding plane in IEEE 802.15.4 domain. Similarly, centralised management is implemented using ip route tool, which allows collaborative routing between heterogeneous nodes within IP layer. The path selection is based on holistic view and directed spatial routing which instructs packets to reach the edge nodes in north-eastern (AP) and north-western (RN) corners of IEEE 802.11g and IEEE 802.15.4 domains respectively. The centralised implementation uses layer 3 as data-forwarding plane in both the domains.

\section{RESUlTS AND DisCUSSION}

We have analysed the property of network interoperability through measure of various network performance metrics, under the schemes of distributed and centralised managements in IoT hetnets. The simulation was conducted under varying networking conditions like: (i) increasing node density (to investigate network scalability); and (ii) degrading link quality (to investigate network reliability). Linux tools iperf and ping are used to measure the performance metrics such as latency, packet loss rate and throughput. To make in-depth analysis, it is necessary to completely study the network operations between initial and converged states of network. Through several iterations, we could observe that simulation duration of 600 seconds and data transmission rate of $1 \mathrm{pkt} / \mathrm{sec}$ were ideal to conduct the experiments. The data transmissions have always been considered between farthest nodes in the simulation as depicted in Fig. 2 .

Most of the studies presented in the related works have ignored the scalability aspects during evaluation, which is an important aspect in large IoT networks [13][14][15]. To include scalability aspects, we consider a maximum of 30 nodes in IEEE 802.11g domain and 30 nodes in IEEE 802.15.4 domain for network setup. For best-effort simulation of nondeterministic network behaviour observed in low-power devices, we have devised link quality degradation or linkfailure by randomly decreasing the probability for successful transmissions (packet drops), where the decrease is bounded within a specified value. Maximum of $10 \%$ packet drop can be supported, before the schemes halt networking when most of the nodes are turned off.

Impact on latency: Latency has been measured as the mean value for round trip time observed in each data transmission. This measurement can been performed only for successful 
transmissions. We can observe a significant difference in latencies under different schemes for network management. Fig. 3 shows that distributed scheme results in latencies from $16.5 \mathrm{~ms}$ up to 2.2 seconds when the total number of nodes in hetnet is ranging from 10 to 60 . This indicates that the scalability has direct and significant impact on network complexity when forming routing trees in distributed management. However, on employing a centralised scheme reveals the latencies are within a range of 20 to $43 \mathrm{~ms}$, indicating that the scheme is more resilient towards scaling networks.

Under varying rates of link-failure through simulated packet drops, Fig. 4 shows that distributed management results in latencies in the range of $2-5$ seconds with 60 nodes in total, while the centralised management requires only few tens of milliseconds. Centralised scheme has lower and more uniform latencies, since the routing graph is diligently built inside the dedicated setup with holistic view of network. In contrast, distributed scheme spends significant time on network convergence or formation of routing graphs, which is formed through independent and best-effort computations of each node. Fig.4a shows an improvement in latencies from $3 \%$ till $10 \%$ with distributed management. This happens at higher drop-rates, as with many nodes duty-cycling, the contentionfree medium allows sporadic bursts in data communication.

Impact on packet loss rate: Loss rate is the ratio of unsuccessful transmissions to the total transmissions (600 in our simulations). Fig.5a show that, inside a lossless environment without packet drops, centralised management is highly reliable when operating against increasing network size. Increasing the number of nodes from 10 to 60 apparently has no significant impact on packet losses with centralised management, while we observe 7 to $25 \%$ packet losses when operating distributed management. With distributed management, a significant outage of network services leading to packet losses is experienced before the network is converged. This convergence period or network outage is directly proportional to the network size. Fig.5b shows that the reduction in link quality of the network causes packet losses under both schemes. Here, both of them lose their operation without routing support from minimal number of active nodes. It means that in heterogeneous IoT networks with lossy links, deteriorating link-quality results in high packet losses or unreliability regardless of the management scheme. Our simulations reveal packet losses from $20-90 \%$ when varying the drop-rate between $1-10 \%$.

Impact on throughput: Throughput is calculated as the mean rate for transmission of largest protocol data unit of internet layer or simply the largest packet (here it is 1240 Bytes). In Fig.6, we observe that the throughput is vastly decreasing in distributed management and has consistent value between 220 and $500 \mathrm{~kb} / \mathrm{s}$ in centralised management with scaling network. This phenomenon is due to the usage of prioritised flow in centralised management which only allows communicating and intermediate nodes to transmit, opposed to distributed management which does not offer any prioritisation. Fig.7 shows with varying drop-rates $(1-10 \%)$, distributed management achieves a mere throughput of $3 \mathrm{~kb} / \mathrm{s}$, while centralised management approximately offers 66 times higher value. This happens because centralised scheme uses active nodes to forward alone, thus, fewer nodes suffice. However, distributed scheme is dependent on the active nodes

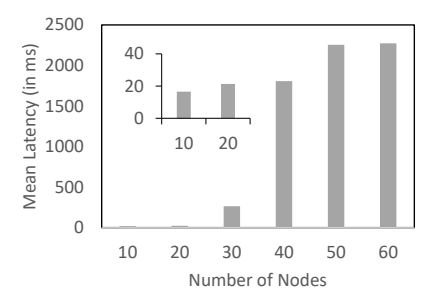

(a)

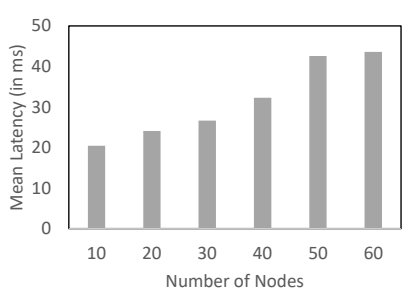

(b)
Fig. 3: Mean latency (in milliseconds) vs number of nodes in (a) distributed management and (b) centralized management

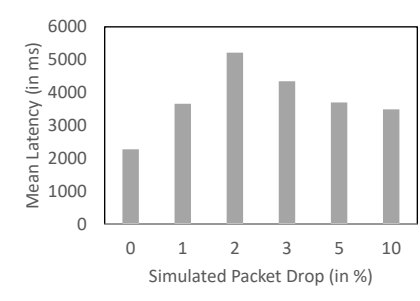

(a)

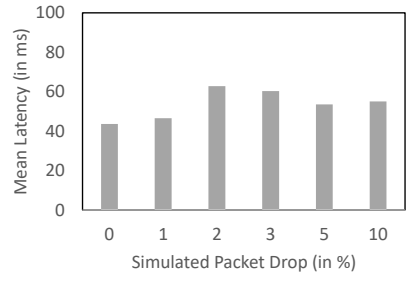

(b)
Fig. 4: Mean latency (in milliseconds) vs packet drop (in \%) in (a) distributed management and (b) centralized management

to compute best route and then forward, that is, large number of duty-cycling nodes affects its operation.

Impact on determinacy: Determinacy is defined as the state of attaining probable performance under varying network dynamics in the hetnet. The state can be analysed by observing the range of variations in network performance namely latency, packet loss rate or throughput. We have considered the latency variations in assessing network determinacy, since latency has the widest dataset from the evaluation. The standard deviation, a statistical method to quantify the fluctuations or dispersion in a dataset, is measured over latency. In table I, minimum, maximum, and mean standard deviations have been listed for different management schemes. Minimum and maximum values give the extent of variations in latency. Here, the mean standard deviation derives the determinacy of each management scheme, that is, smaller mean indicates more consistency in latency irrespective of network dynamics. Evidently, centralised scheme has stood-out as the better approach in terms of deterministic network performance. Given the the collaborative and dedicated effort of centralised management, the network performance is deterministic in wide range of network scalability and dynamics.

Based on our evaluations, we claim that distributed scheme is a better approach for smaller networks with low-cost and self-organised networking, but not preferred for larger networks. The efficiency in centralised management is achieved at the expense of dedicated architecture and holistic view inside network. SDN is a better approach for centralised management and highly programmable network [15]. With SDN in massive hetnets, there is a trade-off between optimisation and operational overhead, in terms of, systemic resources. Nevertheless, with respect to future IoT hetnets, exclusive abilities and stability through centrally managed network clearly outweighs the overhead involved. 


\begin{tabular}{rrrr}
\hline Standard & \multicolumn{2}{c}{ Management schemes } & Occurrence \\
\cline { 2 - 3 } Deviation & Distributed & Centralized & \\
\hline Minimum & 4.755 & 10.154 & With 10 nodes and 0\% packet drop \\
Maximum & 18001.970 & 253.132 & With 60 nodes and 2\% packet drop \\
Mean & 8857.331 & 52.073 & \\
\hline
\end{tabular}

TABLE I: Summary of standard deviation of latencies (in milliseconds).

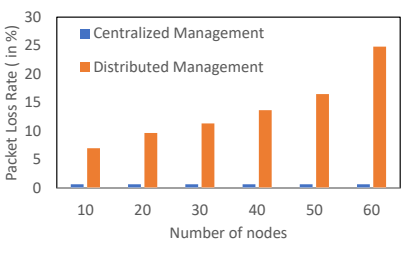

(a)

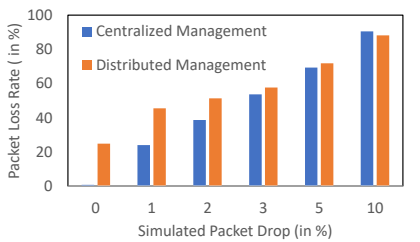

(b)
Fig. 5: Packet loss rate (in \%) under (a) varying number of nodes and (b) varying packet drop (in \%)

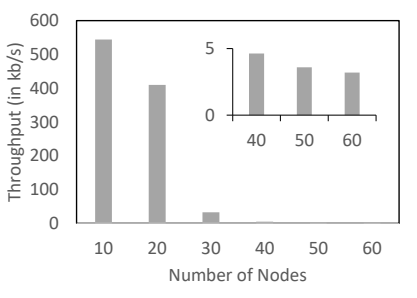

(a)

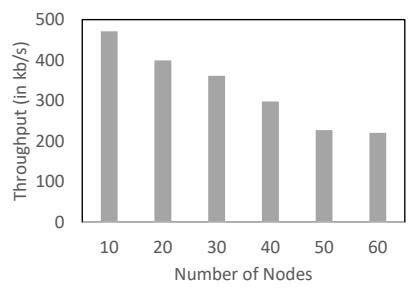

(b)
Fig. 6: Throughput (in $\mathrm{kb} / \mathrm{s}$ ) vs number of nodes in (a) distributed management and (b) centralized management

\section{CONClusion}

Network management focusing interoperability in IoT hetnets can assure QoS of overall network, in terms of connectivity, reachability and scalability. In this work, we implemented a simulation environment, and evaluated distributed and centralised management of network. In our evaluations, the centralised management has excelled at the expense of collaborative effort and dedicated facilities for networking. With usage of scalable industrial Linux kernel in the implementation, the simulation environment can support virtually limitless number of nodes and replicate massive hetnets of the future. In continuation, SDN-based network interoperability in IoT hetnets will be modelled with actual development of centralised manager. Also, the realism in the environment could be improved through better radio interference modelling with the help of supervised machine learning.

\section{REFERENCES}

[1] T. Qiu, N. Chen, K. Li, M. Atiquzzaman, and W. Zhao, "How can heterogeneous internet of things build our future: A survey," IEEE Communications Surveys \& Tutorials, vol. 20, no. 3, pp. 2011-2027, 2018.

[2] H. Fotouhi, A. Causevic, K. Lundqvist, and M. Björkman, "Communication and security in health monitoring systems-a review," in 2016 IEEE COMPSAC, vol. 1. IEEE, 2016, pp. 545-554.

[3] G. Shi and K. Li, Signal interference in WiFi and ZigBee networks. Springer, 2017.

[4] F. X. Wibowo, M. A. Gregory, K. Ahmed, and K. M. Gomez, "Multidomain software defined networking: research status and challenges," Journal of Network and Computer Applications, vol. 87, pp. 32-45, 2017.

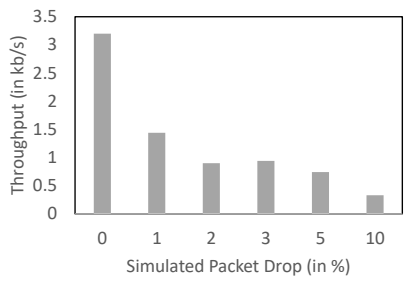

(a)

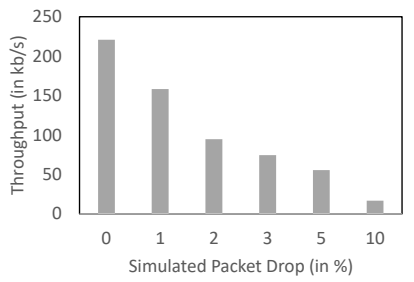

(b)
Fig. 7: Throughput (in $\mathrm{kb} / \mathrm{s}$ ) vs packet drop (in \%) in (a) distributed management and (b) centralized management

[5] A. Geraci, F. Katki, L. McMonegal, B. Meyer, J. Lane, P. Wilson, J. Radatz, M. Yee, H. Porteous, and F. Springsteel, IEEE standard computer dictionary: Compilation of IEEE standard computer glossaries. IEEE Press, 1991.

[6] A. K. Y. Wong, P. Ray, N. Parameswaran, and J. Strassner, "Ontology, mapping for the interoperability problem in network management," IEEE Journal on selected areas in Communications, vol. 23, no. 10, pp. 2058-2068, 2005.

[7] M. Ndiaye, G. P. Hancke, and A. M. Abu-Mahfouz, "Software defined networking for improved wireless sensor network management: A survey," Sensors, vol. 17, no. 5, p. 1031, 2017.

[8] T. De Schepper, P. Bosch, E. Zeljković, F. Mahfoudhi, J. Haxhibeqiri, J. Hoebeke, J. Famaey, and S. Latré, "Orchestra: enabling intertechnology network management in heterogeneous wireless networks," IEEE Transactions on Network and Service Management, vol. 15, no. 4, pp. 1733-1746, 2018.

[9] T. Luo, H.-P. Tan, and T. Q. Quek, "Sensor openflow: Enabling software-defined wireless sensor networks," IEEE Communications letters, vol. 16, no. 11, pp. 1896-1899, 2012.

[10] A. Dunkels, B. Gronvall, and T. Voigt, "Contiki-a lightweight and flexible operating system for tiny networked sensors," in 29th annual IEEE international conference on local computer networks. IEEE, 2004, pp. 455-462.

[11] Mininet-wifi I emulation platform for software-defined wireless networks. [Online]. Available: https://mininet-wifi.github.io/

[12] A. S. D. Alfoudi, S. S. Newaz, R. Ramlie, G. M. Lee, and T. Baker, "Seamless mobility management in heterogeneous 5g networks: A coordination approach among distributed sdn controllers," in 2019 IEEE 89th Vehicular Technology Conference (VTC2019-Spring). IEEE, 2019, pp. 1-6.

[13] K. Sood, K. K. Karmakar, S. Yu, V. Varadharajan, S. R. Pokhrel, and Y. Xiang, "Alleviating heterogeneity in sdn-iot networks to maintain qos and enhance security," IEEE Internet of Things Journal, vol. 7, no. 7, pp. 5964-5975, 2019.

[14] T. Pham, J. McMahon, and H. Nguyen, "Enabling heterogeneous 5g simulations with sdn adapters," in International Conference on Testbeds and Research Infrastructures. Springer, 2019, pp. 163-183.

[15] S. Bera, S. Misra, S. K. Roy, and M. S. Obaidat, "Soft-wsn: Softwaredefined wsn management system for iot applications," IEEE Systems Journal, vol. 12, no. 3, pp. 2074-2081, 2018.

[16] A. G. Anadiotis, S. Milardo, G. Morabito, and S. Palazzo, "Toward unified control of networks of switches and sensors through a network operating system," IEEE Internet of Things Journal, vol. 5, no. 2, pp. 895-904, 2018.

[17] Rpl: Ipv6 routing protocol for low-power and lossy networks. [Online]. Available: https://tools.ietf.org/html/rfc6550

[18] G. R. Hiertz, D. Denteneer, S. Max, R. Taori, J. Cardona, L. Berlemann, and B. Walke, "Ieee 802.11 s: the wlan mesh standard," IEEE Wireless Communications, vol. 17, no. 1, pp. 104-111, 2010.

[19] M. Kerrisk, The Linux programming interface: a Linux and UNIX system programming handbook. No Starch Press, 2010. 